To the Editors:

\title{
Re: HLA-B27 allele frequency in Sri Lankan patients with spondyloarthritides
}

\section{S A S Goonawardena ${ }^{1}$}

Ceylon Medical Journal 2016; 61: 202

10.4038/cmj.v61i4.8397

I read with interest the article by Kidnapillai et al, where the authors found that the frequency of HLA-B27 allele in Sri Lankan patients with spondyloarthritides (SA) to be $22.8 \%$ [1]. However, this article regrettably, leaves a host of questions unanswered by the authors.

- In how many institutions was the HLA-B27 genotype detection conducted?

- What was the gender and age distribution of 373 SA patients?

- What percentage of SA patients among the 373 SA patients were undifferentiated SA, ankylosing spondylitis (AS), psoriatic arthritis (PA), reactive arthritis (RA), Crohn's disease and so on?

- If the sample of 373 SA had only a few AS patients wouldn't it reduce the overall frequency of HLAB27 alleles?

- Do the authors acknowledge that the sample is not representative of Sri Lankan patients with SA?

1 Urology Unit, National Hospital of Sri Lanka, Sri Lanka.

Correspondence: SASG, e-mail: <drsasgurol@yahoo.com>.
HLA genotypetesting is not available in the state sector of Sri Lanka where the healthcare is free of charge. Currently this test costs around SLR 5500 at the Asiri Center for Genomic and Regenerative Medicine at Asiri Surgical Hospital, Colombo.

In view of the ability to afford such a costly test or otherwise only a select group of SA patients would enter the database for HLA-B27 testing. Hence the title of this article "HLA-B27 allele frequency in Sri Lankan patients with spondyloarthritides" is misleading. If a sizeable sample of consecutive patients with SA in a busy rheumatology clinic in a state hospital is studied a more accurate picture of the HLA-B27 allele frequency could be obtained that can be construed as representative of Sri Lankan patient with SA.

\section{Reference}

1. Kidnapillai AS, Sirisena ND, Dissanayake VHW. HLA-B27 allele frequency in Sri Lankan patients with spondyloarthritides Ceylon Med J 2016; 61: 71-3. 